\title{
Análisis crítico de un artículo: ¿Es seguro no tratar a pacientes con sospecha de tromboembolismo pulmonar y tomografía axial computarizada negativa?
}

\author{
Juan Abarza ${ }^{1}{ }^{a}$, G abriel Rada ${ }^{2,3}$. \\ Critically appraised article: \\ Is it safe not to treat patients \\ with pulmonary embolism \\ and a negative $C T$ scan? \\ Quiroz R, Kucher N, Zou K, \\ Kipfmueller F, Costello P, Goldhaber \\ S, Schoepf UJ. JAMA 2005; 293: \\ 2012-7.
}

\begin{abstract}
Context: The clinical validity of using computed tomography (CT) to diagnose peripheral pulmonary embolism is uncertain. Insufficient sensitivity for peripheral pulmonary embolism is considered the principal limitation of CT. Objective: To review studies that used a CT-based approach to rule out a diagnosis of pulmonary embolism. Data sources: The medical literature databases of PubMed, MEDLINE, EMBASE, CRISP, metaRegister of Controlled Trials, and Cochrane were searched for articles published in the English language from January 1990 to May 2004. Study selection: We included studies that used contrastenhanced chest CT to rule out the diagnosis of acute pulmonary embolism, had a minimum follow-up of 3 months, and had study populations of more than 30 patients. Data extraction: Two reviewers independently abstracted patient demographics, frequency of venous thromboembolic events (VTEs), CT modality (single-slice CT, multidetector-row CT, or electronbeam CT), false-negative results, and deaths attributable to pulmonary embolism. To calculate the overall negative likelihood ratio (NLR) of a VTE after a negative or inconclusive chest CT
\end{abstract}

\footnotetext{
${ }^{1}$ Servicio de Medicina, Hospital de Urgencia Asistencia Pública, Santiago, Chile. ${ }^{2}$ Unidad de Medicina Basada en Evidencia, Pontificia Universidad Católica de Chile. Santiago, Chile. ${ }^{3}$ Departamento de Medicina Interna, Pontificia Universidad Católica de Chile. Santiago, Chile.

${ }^{\text {aResidente }}$
} 
scan for pulmonary embolism, we included VTEs that were objectively confirmed by an additional imaging test despite a negative or inconclusive CT scan and objectively confirmed VTEs that occurred during clinical follow-up of at least 3 months. Data synthesis: Fifteen studies met the inclusion criteria and contained a total of 3500 patients who were evaluated from October 1994 through April 2002. The overall NLR of a VTE after a negative chest CT scan for pulmonary embolism was 0.07 (95\% confidence interval [CI], 0.05-0.11); and the negative predictive value (NPV) was 99.1\% (95\% CI, 98.7\%-99.5\%). The NLR of a VTE after a negative single-slice spiral CT scan for pulmonary embolism was 0.08 (95\% CI, 0.05-0.13); and after a negative multidetector-row CT scan, 0.15 (95\% CI, 0.05-0.43). There was no difference in risk of VTEs based on CT modality used (relative risk, 1.66; 95\% CI, 0.47-5.94; $\mathrm{P}=.50$ ). The overall NLR of mortality attributable to pulmonary embolism was 0.01 (95\% CI, 0.01-0.02) and the overall NPV was 99.4\% (95\% CI, 98.7\%-99.9\%). Conclusion: The clinical validity of using a CT scan to rule out pulmonary embolism is similar to that reported for conventional pulmonary angiography.

\section{CONCLUSIÓN DE LOS REVISORES:}

La tomografía axial computarizada (TAC) negativa para tromboembolismo pulmonar (TEP) es útil para descartar un TEP clínicamente relevante.

\section{Pregunta CĹNICA:}

En pacientes con sospecha de tromboembolismo pulmonar dia tomografía axial computarizada permite descartar un tromboembolismo pulmonar clínicamente relevante?

\section{ConTEXTO:}

El uso de la TAC pulmonar en el diagnóstico del tromboembolismo pulmonar se ha hecho cada vez más frecuente. Su cada vez mayor disponibilidad y su buena visualización del parénquima pulmonar han contribuido a esto.

Existe discusión en la literatura acerca de qué método es el más adecuado para el diagnóstico de TEP, ya que la TAC tiene sensibilidad insuficiente para detectar émbolos en los segmentos periféricos de la vasculatura pulmonar ${ }^{1}$. Si bien la angiografía se ha considerado el estándar de oro para el diagnóstico de TEP, los distintos estudios muestran una importante variabilidad interobservador ${ }^{2}$. Por otra parte, aunque se lograra hacer un diagnóstico certero de los TEP periféricos, la relevancia clínica de éstos no está clara.

Una alternativa a la angiografía u otros métodos de imágenes, para evaluar el rendimiento diagnóstico de la TAC, es la observación en el tiempo de la ausencia de enfermedad tromboembólica (ETE). En términos simples, si un paciente en quien se sospechó un TEP con TAC negativa, no presenta evidencias clínicas de ETE durante el seguimiento, a pesar de no haber sido tratado con anticoagulantes, se podría concluir que nunca la tuvo, o que ésta no fue clínicamente relevante. En esta situación, la utilización de mejores métodos de imágenes no sería necesaria. 


\section{CARACTERÍSTICAS DEL ESTUDIO:}

Tipo de estudio: Revisión sistemática de estudios de diagnóstico. La búsqueda se realizó en 6 bases de datos electrónicas, incluyendo MEDLNE y EMBASE, hasta mayo de 2004. Se realizó una búsqueda manual en revistas seleccionadas y en las referencias de los estudios encontrados. Se contactó a autores y a expertos en el área.

Se incluyeron estudios que evaluaran la utilización de TAC con contraste en pacientes con sospecha de TEP. Se incluyeron estudios utilizando cualquier modalidad de TAC. Además los estudios debían tener un seguimiento mínimo de 3 meses, un método adecuado de seguimiento, más de 30 pacientes y la TAC debía haberse realizado en todos.

Para calcular el likelihood ratio negativo (LR para TAC negativa) y valor predictivo negativo (VPN), se consideraron los pacientes en que se diagnosticó TVP durante el episodio que motivó la TAC (por métodos de imágenes adicionales), y los episodios de TVP/TEP con manifestaciones clínicas durante el seguimiento (confirmados por imágenes). En otras palabras, el test en este caso es la TAC y el estándar de oro es algún evento tromboembólico que requiera anticoagulación» que la TAC no hubiese detectado, ya sea una TVP concomitante o una TVP/TEP durante el seguimiento. Adicionalmente, se calculó el LR y VPN utilizando la mortalidad por TEP como estándar de oro.

VALDEZ:

\begin{tabular}{|ll|}
\hline Pregunta específica y focalizada & Sí \\
Búsqueda amplia y completa & Parcialmente* \\
Criterios de inclusión explícitos y adecuados & Sí \\
Evaluación de calidad de los estudios incluidos & Sí** \\
Reproducibilidad del proceso & Sí \\
Evaluación de heterogeneidad & Sí \\
\hline
\end{tabular}

* Sólo se incluyeron estudios en idioma inglés. No se buscó en resúmenes de congresos. * Se utilizó un score de calidad creado por los autores que no incluye los criterios clásicos de validez de un estudio diagnóstico.

\section{ResUlTADOS:}

Se incluyeron 15 estudios. En total, 3.500 pacientes tuvieron un resultado negativo para TEP y se consideraron en la revisión. La prevalencia de TEP en los estudios fue de 15\% a $38 \%$. Doce estudios utilizaron TAC de un detector, 2 utilizaron TAC con multidetector y uno utilizó TAC de haz de electrones (electron-beam CT). El tiempo de seguimiento fue de $3 \mathrm{a}$ 12 meses. Se realizó metaanálisis utilizando modelo de efectos aleatorios, el cual no detectó heterogeneidad en los estudios incluidos. 
Tabla 1. Resultados de la revisión sistemática

\begin{tabular}{|lcc|}
\hline Estándar de oro & $\begin{array}{c}\text { LR para TAC negativa } \\
\text { (IC 95\%) }\end{array}$ & $\begin{array}{c}\text { VPN } \\
\text { (IC 95\%) }\end{array}$ \\
\hline Evento tromboembólico & 0,07 & $99,1 \%$ \\
Mortalidad & (IC: $0,05-0,11)$ & (IC: $98,7-99,5 \%)$ \\
& 0,01 & 99,4 \\
& (IC: $0,01-0,02)$ & (IC: $98,7-99,9)$ \\
\hline
\end{tabular}

LR =Likelihood Ratio (Razón de probabilidad), VPN =Valor predictivo negativo, IC $=$ Intervalo de confianza.

Tabla 2. Probabilidad de evento tromboembólico en pacientes con TAC negativa

\begin{tabular}{|lcc|}
\hline Probabilidad pretest* & $\begin{array}{c}\text { Likelihood ratio } \\
\text { para TAC negativa** }\end{array}$ & Probabilidad postest \\
\hline Baja (10\%) & 0,07 & $1 \%$ \\
Intermedia (30\%) & 0,07 & $3 \%$ \\
Alta (70\%) & 0,07 & $14 \%$ \\
\hline
\end{tabular}

Basado en probabilidades aproximadas según score de Wells para TEP ${ }^{3}$.

* LR para predecir eventos tromboembólicos en pacientes con TAC negativa. Asumiendo que LR es constante en pacientes con distinta probabilidad pretest.

\section{COMENTARIOS Y APLICACIÓN PRÁCTICA:}

* El estudio cumple parcialmente con los criterios de validez de una revisión sistemática. La escala utilizada en la evaluación de los estudios incluidos no permite hacerse una clara idea de la calidad de éstos.

* Dentro de los exámenes utilizados para el diagnóstico de TEP durante el seguimiento se utilizó frecuentemente la TAC. Esto podría constituir una amenaza a la validez, debido a que no existe independencia entre el test y el estándar. En un paciente con TEP que no hubiese sido detectado por la TAC (falso negativo), si al presentar un nuevo evento, se realizó el mismo examen como método confirmatorio, podría volver a repetirse el error diagnóstico, introduciendo sesgo.

* La utilización de outcomes clínicos como el estándar de oro, constituye una manera distinta de aproximarse al problema de la importancia clínica del TEP que compromete sólo segmentos periféricos de la vasculatura pulmonar. En términos simples, responde a la pregunta, cpuede la TAC descartar un TEP que tenga alguna repercusión clínica? Al considerar esto, hay que tener en cuenta que 199 pacientes (6\%) no pudieron ser seguidos (no se supo si tenían TVP o TEP). Por otro lado, la evaluación de la mortalidad por TEP puede ser subestimada, por las dificultades diagnósticas en esta patología, por lo que hubiese sido importante la evaluación de la mortalidad total ${ }^{4}$.

* El tiempo de seguimiento exigido parece razonable, especialmente considerando que la mayoría de los eventos que podrían no ser detectados ocurren en un corto período ${ }^{5}$. La observación por periodos largos podría detectar eventos nuevos, sin relación con el 
cuadro que motivó la TAC. Esto puede haber ocurrido en estos estudios, sin embargo parece razonable asumir una posición más conservadora.

* La probabilidad pretest de los distintos estudios cae en el rango de moderada. No puede extrapolarse a pacientes con probabilidad pretest alta, en quienes la probabilidad postest puede no ser suficientemente baja como para prescindir de la anticoagulación (Tabla 2). En pacientes con probabilidad baja, podrían utilizarse estrategias diagnósticas de menor costo y riesgo, como el dímero $\mathrm{D}$, aunque no permite descartar el diagnóstico en una proporción importante de los pacientes, en quienes muy probablemente se realizará una $\mathrm{TAC}^{6}$.

* No se realiza un análisis del rendimiento de las TAC no diagnósticas, que correspondieron a $153(4 \%)$.

* Una duda que se plantea, a la luz de esta revisión sistemática es si la mejoría en las técnicas radiológicas permite detectar con mayor certeza TEP clínicamente significativos, o sólo detecta aquellos que no tienen importancia para el paciente. En esta revisión, la TAC de un detector fue la más utilizada y no mostró diferencias con otras modalidades. Por otra parte, el tratamiento anticoagulante no está exento de riesgos, por lo que sería deseable que futuros estudios evalúen en forma clara el riesgo y el beneficio de tratar a pacientes con TEP pequeños o periféricos.

* En conclusión, no sería necesario realizar estudio adicional en pacientes con probabilidad pretest moderada para TEP que tienen una TAC negativa y sería seguro no iniciarles tratamiento anticoagulante. Esta conducta permitiría evitar los costos y riesgos de la TAC o de otros procedimientos diagnósticos.

\section{REFERENCIAS}

1. Rathbun SW, RasKob GE, Whitsett TL Sensitivity and specificity of helical computed tomography in the diagnosis of pulmonary embolism: a systematic review. Ann Intern Med 2000; 132: 227-32.

2. Ruiz Y, Cabalero P, Caniego JL. Prospective comparison of helical CT with angiography in pulmonary embolism: global and selective vascular territory analysis: interobserver agreement. Eur Radiol 2003; 13: 823-9.

3. Wells PS, Anderson DR, Rodger M. Derivation of a simple clinical model to categorize patients' probability of pulmonary embolism: increasing the model's utility with the SimpliRED D-dimer. Thromb Haemost 2000; 83: 416-20.

4. Stein PD, TerRin ML, Haies CA. Clinical, laboratory, roentgenographic, and electrocardiographic findings in patients with acute pulmonary embolism and no preexisting cardiac or pulmonary disease. Chest 1991; 100: 598-603.

5. Carson JL, Keley MA, DufF A. The clinical course of pulmonary embolism. NEngl J Med 1992; 326: $1240-5$.

6. Welis PS, Anderson DR, Rodger M. Evaluation of d-dimer in the diagnosis of suspected deepvein thrombosis. N Engl J Med 2003; 349: 1227-5. 